\title{
Sitio web para incentivar el hábito lector en los estudiantes que van a ingresar a la Universidad
}

Enviado: 16 de diciembre de 2020 / Aceptado: 3 de marzo de 2021 / Publicado: 12 de julio de 2021

TANIA CATALINA HURTADO

taniahurtado86@hotmail.com

PAUL BALDEÓN EGAS

Universidad Israel, Ecuador pbaldeon@uisrael.edu.ec

\section{RESUMEN}

La lectura es una de las actividades de mayor importancia para la aprehensión del conocimiento. El objetivo de la presente investigación es incentivar el hábito lector, mediante el uso de un sitio web, elaborado en base a las necesidades y sugerencias de los estudiantes de nivelación de carrera de la Universidad Central del Ecuador. Las metodologías a ser utilizadas, dentro de los bloques académicos, son PACIE y ERCA aplicando en cada una de ellas actividades que sean interactivas. Se determinó que, en base a una mejor estructuración del tiempo de los estudiantes, se puede fomentar el hábito lector usando la página web.

Palabras Clave: hábito lector, TIC, estudiantes universitarios.

\section{ABSTRACT}

Website to Encourage the Reading Habit in Students that are going to go to the University

Reading is one of the most important activities for the apprehension of knowledge. The objective of this research is to encourage the reading habit through the use of a website developed based on the needs and suggestions of the students of Career Leveling of Universidad Central del Ecuador. The methodologies to be used, in the academic blocks, are PACIE and ERCA, applying activities that are interactive to each of them. It was determined that, based on a better structuring of student's time, the reading habit can be promoted using the website.

Keywords: reading habit, ICT, college students. 


\section{INTRODUCCIÓN}

Según un estudio realizado por el Diario Semana (2017) "la mayoría de niños y adolescentes en Latinoamérica carecen de competencias básicas en lo que a lectura corresponde, considerando esto en una situación dramática”. Por lo tanto, se debe tener en cuenta que esta es una habilidad fundamental, sobre la cual se siguen construyendo las demás destrezas cognitivas.

En el Ecuador, la lectura es una de las más grandes barreras en el desarrollo cultural del mismo. Debido a que aún en el país se sostiene un modelo educativo tradicional, en donde predomina como herramienta de estudio el memorizar; existiendo así, poca reflexión. El factor económico es relevante debido a que los estudiantes destinan el tiempo libre a generar recursos, quedando actividades como la lectura relegadas a un segundo plano y afectando en su rendimiento educativo.

El Instituto Nacional de Estadísticas y Censos (INEC) menciona que "el 27\% de los ecuatorianos no tiene el hábito de la lectura. Para el INEC, las razones principales son la falta de interés que corresponde al 56,8\% y la falta de tiempo" (INEC, 2013). Además de lo antes mencionado, el uso inadecuado de la tecnología es otra de las razones por las cuales los ecuatorianos no poseen un hábito de lectura.

Por lo tanto, en el presente estudio, se pretende determinar aquellos mecanismos que faciliten el desarrollo del hábito lector en los estudiantes de la Universidad Central del Ecuador.

\section{MATERIAL Y MÉTODO}

Se ha determinado, en primer lugar, el problema de partida de la investigación para luego fundamentar los objetivos que serán aquellos que se buscan alcanzar al finalizar la misma.

El presente proyecto es de carácter mixto. Cedeño (2012) menciona que dichos tipos de proyectos son una "investigación en la que los métodos mixtos representan un conjunto de procesos sistemáticos, empíricos y crítico de investigación e implican la recolección y el análisis de datos cuantitativos y cualitativos, así como su integración y discusión conjunta”. Se ha seleccionado a este tipo de investigación con la finalidad de realizar deducciones de toda la información obtenida, logrando así un mayor entendimiento de lo investigado. 
En lo que respecta a la profundidad de la investigación, esta será de tipo exploratorio, debido a que se va a obtener, de manera provisional, conocimientos de la situación. La investigación exploratoria busca una visión general acerca de una determinada realidad y se la realiza sobre temas que han sido poco estudiados.

Al desarrollar la investigación dentro del aula de clases, se define a la misma como una investigación de campo, puesto que, en base a lo que se investigue, se podrá realizar una generalización de los resultados. Así, se extraerán los datos informativos de manera directa de la realidad que va a ser investigada, usando técnicas de recolección de información. Al no tener un control riguroso de los parámetros a investigar, no se puede clasificar al proyecto como una investigación de laboratorio.

González y Gallardo (2007) afirman que los métodos "son el camino, la vía para alcanzar los fines de la investigación". Por tanto, estos deben corresponder de manera directa a las metodologías que se determinaron en un principio, es la manera en la se va a realizar de forma sistemática, organizada la recolección de información. El método de investigación a ser utilizado es inductivo-deductivo porque se inicia analizando los datos para luego generalizarlos. Abreu (2014) menciona que "es importante destacar que el método se deriva de la teoría. Esta selección del método depende de tres elementos: el tipo de fenómeno a estudiar, los objetivos de la investigación y la perspectiva de análisis del investigador”. Lo que implica que el método antes mencionado es el más adecuado para el tipo de investigación a realizarse, permitiendo generalizar los resultados a obtener.

La técnica que va a utilizarse será la encuesta. Según Álvira (2011) "la encuesta es esencialmente una técnica de recogida de información con una filosófica subyacente”, misma que va a ser aplicada a estudiantes, en un primer momento, con el propósito de determinar las razones por las cuales a un alumno no le gusta la lectura o cuál es la razón por la que la lectura no es parte de su rutina diaria. En base a esto se podrá establecer qué actividades serán más adecuadas para fomentar el hábito lector.

La finalidad de la encuesta es recopilar las diversas opiniones que pueden tener los estudiantes de la muestra seleccionada, con el propósito de valorar el indicador planteado.

Los métodos y técnicas utilizados se explican en la siguiente Tabla 1. 
Tabla 1. Aplicación de la tecnología. Fuente: Elaboración propia

\begin{tabular}{|c|c|c|c|}
\hline Métodos y Técnicas & Población & Objetivo & Indicadores a valorar \\
\hline Encuesta & $\begin{array}{l}\text { Estudiantes de Nive- } \\
\text { lación de la Carrera } \\
\text { de Pedagogía de } \\
\text { Idiomas Nacionales } \\
\text { y Extranjeros de la } \\
\text { Universidad Central } \\
\text { del Ecuador. }\end{array}$ & $\begin{array}{l}\text { Conocer las distintas opiniones } \\
\text { referentes a la lectura de los estu- } \\
\text { diantes de Nivelación de la Carrera de } \\
\text { Pedagogía de Idiomas Nacionales y } \\
\text { Extranjeros de la Universidad Central } \\
\text { del Ecuador. }\end{array}$ & $\begin{array}{l}\text { Aplicación de las TIC para } \\
\text { incentivar el hábito lector. }\end{array}$ \\
\hline
\end{tabular}

Es pertinente aclarar que el área de Nivelación se encuentra dividida por carreras. En esta investigación se tomará como población la Carrera de Pedagogía de Idiomas Nacionales y Extranjeros, misma que cuenta con 36 estudiantes pertenecientes, a un mismo paralelo y que cuentan con características similares.

Para determinar la muestra, se realizará un muestreo intencional. Según el sitio web QuestionPro.com (2017) "el muestreo intencional, también conocido como muestreo discrecional o muestreo por juicio, se utiliza para ayudar a responder la pregunta principal de una investigación". Se ha escogido esta modalidad de muestreo debido a que, dentro del grupo de estudiantes, todos poseen características comunes entre sí. Por tanto, y en base al juicio selectivo del docente, se tomó en cuenta a este grupo para el estudio.

Posteriormente, se aplicó la encuesta a los 36 estudiantes de la Carrera de Pedagogía de Idiomas Nacionales y Extranjeros, encuesta que se estableció a manera de diagnóstico.

El fundamento teórico del proyecto se basa en la articulación de los componentes del modelo pedagógico mediado por las Tecnologías de Información y Comunicación (TIC). Por una parte, se encuentra lo teórico, fundamentado en el Constructivismo, donde el individuo al actuar sobre el medio en el que se desenvuelve, construye estructuras en su propia mente. Este modelo fue desarrollado por Piaget y el Desarrollo del Proceso de Aprendizaje, por Ausubel. Se menciona que en un "aprendizaje significativo, los nuevos conocimientos se incorporan en forma sustantiva en la estructura cognitiva del alumno" (Sabori, 2009). Eso quiere decir que será fundamental incentivar el hábito lector en los estudiantes de Nivelación. "El Constructivismo fue el marco teórico que orientó la investigación en el sentido de que contribuyó al análisis, explicación e interpretación del proceso de enseñanza y aprendizaje de la lectura que se desarrolla en la escuela" (Caldera de Briceño et al., 2010). Es importante tener en cuenta el 
proceso que se tiene en cuanto a la enseñanza-aprendizaje en la época escolar, para determinar las fallas en la época universitaria y las posibles correcciones que se pueden lograr.

Por otra parte, se encuentra el Conectivismo que "es definido como una teoría de aprendizaje para la era digital" (Siemens, 2004). Por ello, es importante su implementación dentro del contexto del sitio web desarrollado. Adicionalmente, Hernández (2013) señala que hay que "ampliar nuestra comprensión de las habilidades cognitivas y lingüísticas de humanos y animales y la posibilidad de generar formas artificiales de inteligencia”. De esta manera, el hecho de comprender estas nuevas formas de inteligencia artificial facilitará el trabajo de fomentar la lectura mediante un sitio web, además de combinarlas con metodologías tradicionales, potenciando la manera en la que se imparte el aprendizaje dentro del aula.

En el aspecto metodológico, se desarrolló la estructura PACIE. Según Camacho (2012) dicha estructura "es una metodología que permite el uso de las TIC como un soporte a los procesos de aprendizaje y autoaprendizaje, dando realce al esquema pedagógico de la educación real”. La metodología PACIE será utilizada dentro del Bloque 0, que en la página web se denominó "Sobre mí". Por su parte, Oñate (2009) afirma que "la metodología PACIE es una metodología para el uso y aplicación de las herramientas virtuales (aulas virtuales, campus virtuales, web 2.0, metaversos, etc...) en la educación sea en sus modalidades presenciales, semipresenciales o a distancia”. Al utilizar una página web como herramienta principal y como complemento a Google Classroom, se aplica esta metodología con el fin de facilitar el proceso de generar el hábito lector en los estudiantes.

Mientras que en los siguientes bloques se manejará la metodología ERCA que, en base Rodríguez et al. (2013) es establecer una "secuencia de aprendizaje que oriente la planificación de la formación que favorece la integridad y la coherencia formativa de sus componentes y facilita la interpretación de los logros y las carencias de las unidades formativas una vez implementadas". Aplicando la metodología ERCA se pretende estructurar de mejor manera las actividades que se proponen dentro de la plataforma y poder fomentar el hábito lector. Así, se promoverán de forma contundente, las estrategias utilizadas dentro de la plataforma.

El componente estratégico será el uso de actividades sincrónicas y asincrónicas, utilizadas en la página web: https://taniahurtado.jimdofree.com/

Es importante tener en cuenta algunas consideraciones didácticas al momento de propiciar la lectura. En primer lugar, dentro del bloque PACIE, que en la página web se denomina 
"Sobre mí", se da una introducción de la persona que está a cargo de llevar todo el proceso y la manera en la que se desenvolverán las actividades. En los demás bloques se iniciarán con lineamientos que guiarán al estudiante para realizar las actividades. En dichos bloques se aplicará la metodología ERCA.

Adicionalmente es necesario que la persona que lleve a cargo el proceso proporcione las directrices adecuadas, mismas que deben generar un proceso de reflexión en los estudiantes. En segundo lugar, y antes de iniciar con el desarrollo de incentivar el proceso de lectura, se deben tener claros los objetivos y las estrategias que se utilizarán. Por último, se deben considerar y tener en cuenta las diferentes maneras en las que los estudiantes pueden adquirir los conocimientos.

Las actividades utilizadas en la página son pensadas de tal manera para que, conforme el estudiante las realice, vaya desarrollando el hábito lector. El sitio está estructurado en sesiones y cada una de ellas tiene actividades que motivan y promueven los beneficios de la lectura, para luego pasar a la reflexión, en donde el estudiante va a relacionar su experiencia previa con lo revisado en la página web. A continuación, se realizarán actividades en las que se sistematizarán todos los conceptos adquiridos previamente. Por último, existen actividades donde los estudiantes pueden interactuar y aplicarlas dentro de sus actividades diarias.

En la Figura 1 se evidencia la primera página del sitio web, donde se han creado 3 sesiones y en las cuales se encuentran todas las actividades a desarrollarse: Un apartado para saber sobre la página, otro del blog y finalmente uno de contactos.

Figura 1. Bloques Académicos. Fuente: Elaboración propia

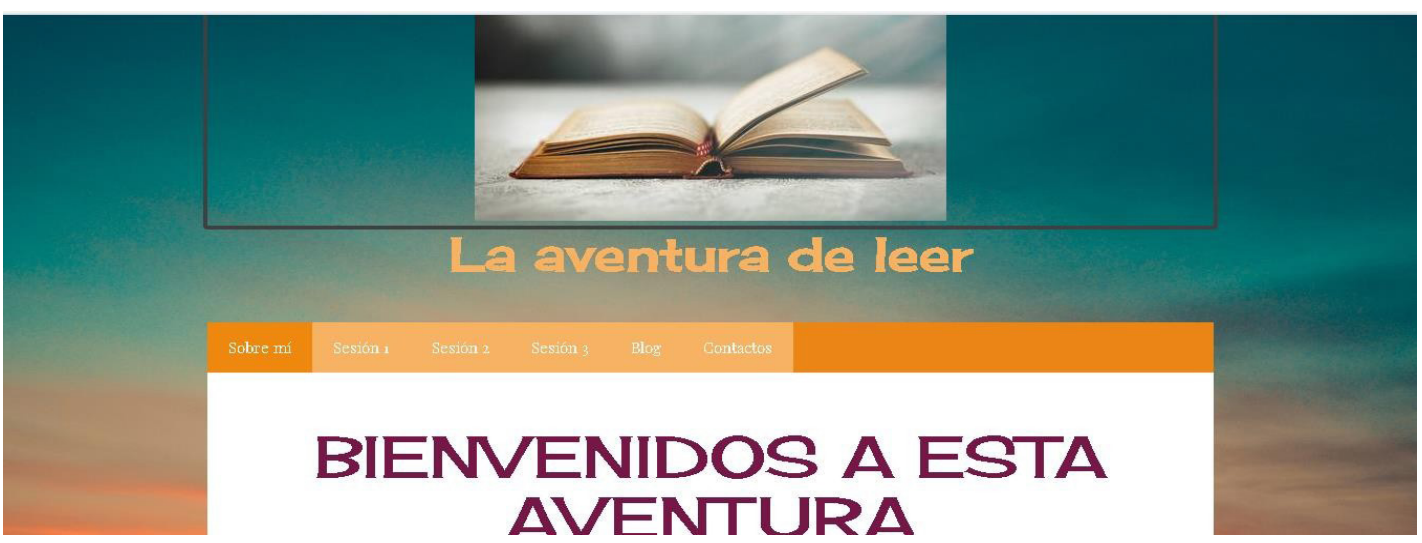

Al ingresar a la sesión 1 (Figura 2) se podrá encontrar un área de reflexión, que invita a conocer los beneficios de la lectura, que se encuentran en infografías. 
Figura 2. Bloque Académico (Sesión 1) - Reflexión. Fuente: Elaboración propia

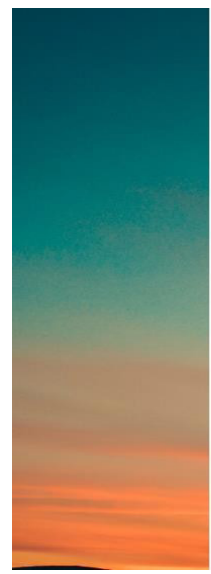

Revisa las imágenes a continuación y prepárate para crear.
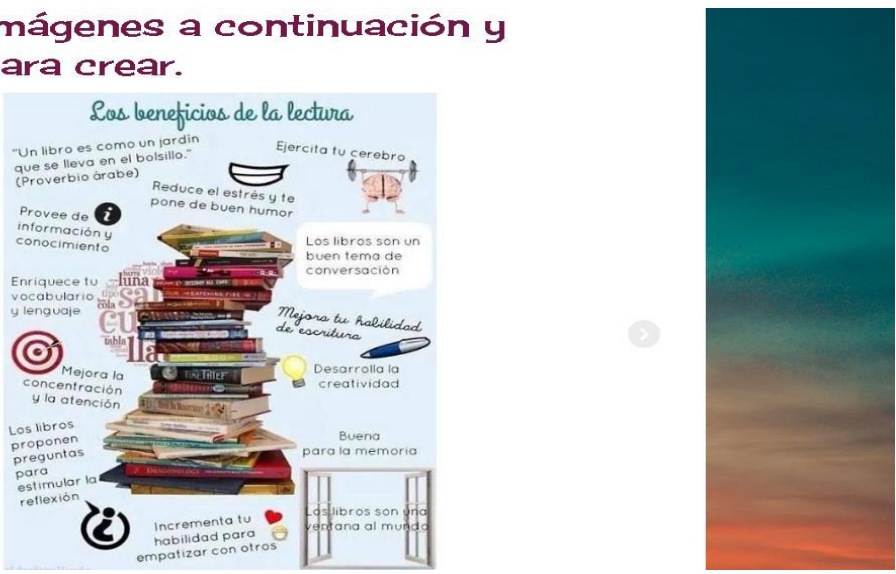

En la Figura 3 se muestra la Sesión 2. Dentro de ella está la primera actividad a realizarse.

Figura 3. Bloque Académico (Sesión 2) - Reflexión. Fuente: Elaboración propia

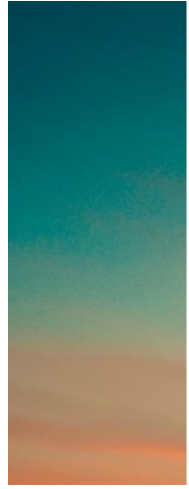

A continuación debes realizar un organizador gráfico como el que esta a continuación, con el tema: "Tipos de lectores"
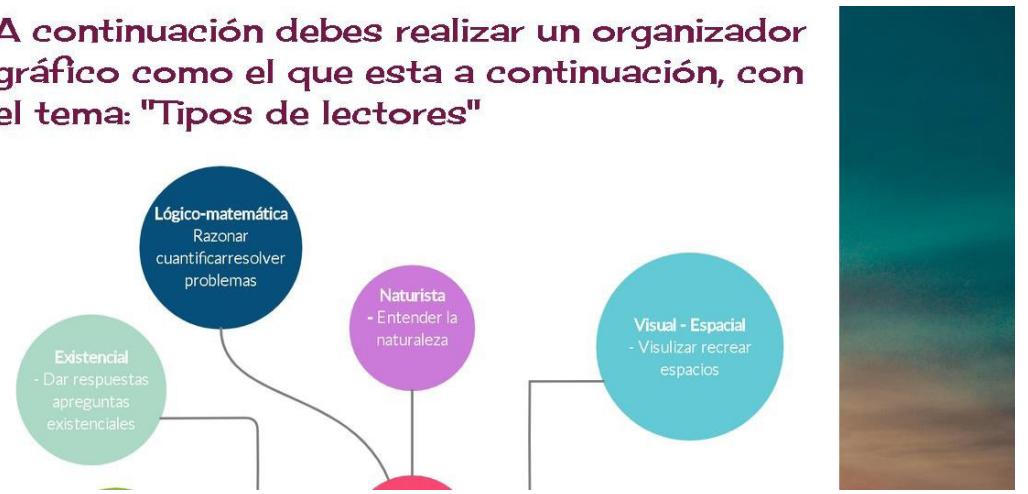

Y en la Figura 4 se evidencia la Sesión 3, con el bloque académico correspondiente.

Figura 4. Bloque Académico (Sesión 3) - Aplicación. Fuente: elaboración propia

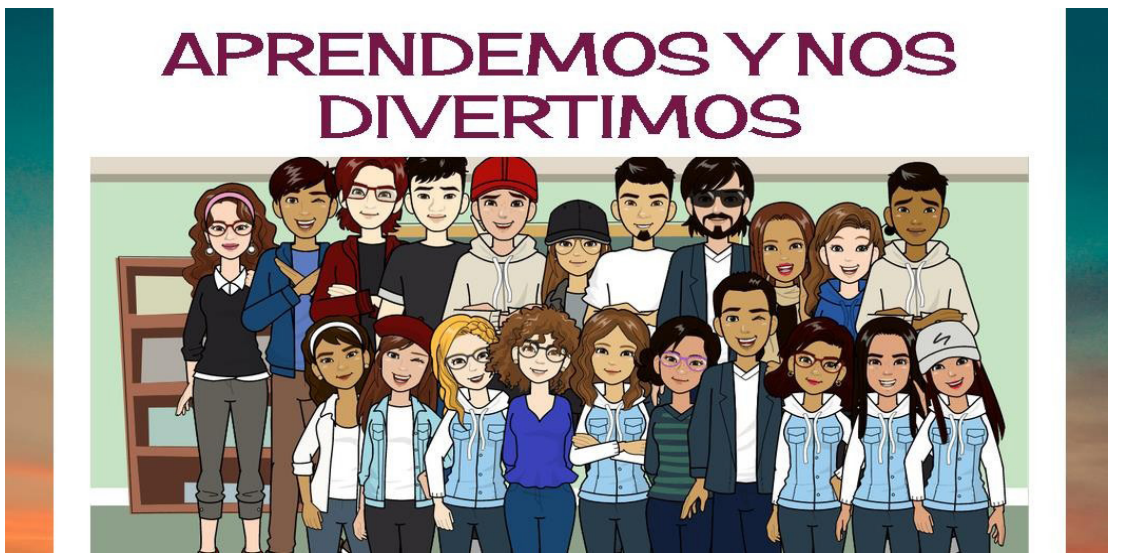


Existen varios estudios que mencionan la importancia de la lectura a lo largo de la vida del ser humano y al ingresar a la universidad se da por hecho que los estudiantes tienen el hábito lector. Carlino (2003) menciona que "es preciso reconceptualizar lo que está en juego cuando los alumnos se enfrentan a comprender los textos que propone la universidad." Los estudiantes que ingresan a la universidad tienen problemas al tratar de comprender los textos que se les indica para su proceso académico. Adicionalmente, Carlino (2005) afirma que se debe "desplegar mayor actividad intelectual que la implicada en escuchar al docente y leer la bibliografía una semana antes del examen parcial." Es importante la lectura en el ámbito académico y sobre todo la comprensión de lo que los estudiantes leen.

Cabe mencionar que el proceso para adquirir un hábito de lectura no es rápido ni inmediato. Dicho proceso "no ocurre de manera espontánea, al contrario, es un proceso continuo y sostenido que requiere acompañamiento lúdico y sistemático, para que pueda mantenerse como un hábito a lo largo de la vida” (León, 2018). Debido a ello, se debe generar actividades que sean atractivas (para captar la atención del estudiante) y fáciles de realizar. Si esto se cumple, no existirá aburrimiento y se generará poco a poco el hábito de la lectura.

Figura 5. Articulación componentes del Modelo Pedagógico mediado por las TIC. Fuente: Elaboración Propia

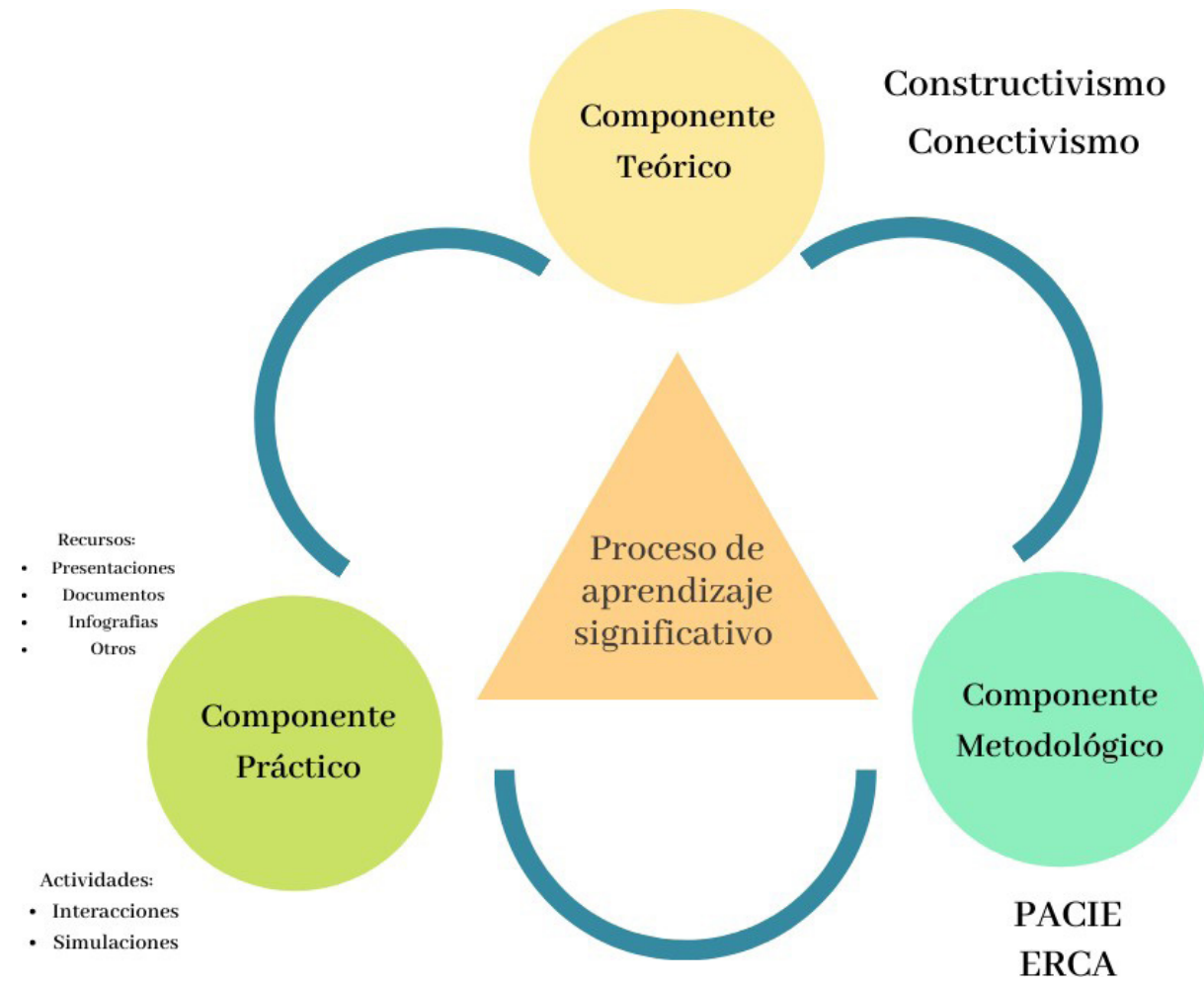


Es fundamental el papel que desarrolla el docente. Trejo \& Alarcón (2006) afirman que "los docentes universitarios debemos conocer, analizar y manejar las estrategias que permiten una eficiente comprensión lectora, implantar los procesos de lectura como una de las funciones básicas de la educación sistemática”. Las estrategias que se utilizan son vitales para fomentar el hábito lector y deben ir encaminadas a la manera en la que cada estudiante pueda percibir de mejor manera los conocimientos.

En la Figura 5 se indica la articulación que existe entre el componente teórico- metodológico y práctico.

\section{RESULTADOS}

Los estudiantes de la Carrera de Pedagogía de Idiomas Nacionales y Extranjeros son conscientes de la necesidad sobre tener un hábito lector y afirmaron que, el hecho de no tenerlo ha sido un impedimento para desenvolverse de manera adecuada en el ámbito educativo. Al aplicar las actividades que se presentaron en el sitio web, los estudiantes determinaron que su interés en la lectura aumenta y que, al tener un cronograma de actividades mejor organizado, leer se convierte en una actividad que es parte de su rutina diaria.

Además, se afirmó que la tecnología es una herramienta útil para incentivar el desarrollo de una cultura lectora. Los estudiantes sugirieron que prefieren realizar actividades de escritura dentro del sitio web JIMDO, plataforma en la cual se insertó un link para una herramienta tecnológica en la que ellos tuvieron espacio para practicar actividades de lectoescritura acordes a su edad. Además, se insertó una pestaña en la cual está el blog, donde después de cada lección los estudiantes escribieron sus impresiones de las actividades realizadas, fomentando, de manera paralela, la lectura y escritura.

Algo que se debe destacar es que, al preguntar qué tipos de lectura prefieren, las respuestas fueron variadas. Es por esta razón que se insertó un link en el cual se tiene libre acceso a un repositorio de libros gratuitos y donde existen diversos títulos que serán de interés para todo tipo de lector. Por lo tanto, se debió tomar en cuenta este factor al momento de implementar dichas actividades dentro del sitio web (Figura 6).

El proyecto está sustentado en una estructura tecno-educativa. Con el fin de aportar al desarrollo del hábito lector se seleccionó a la plataforma JIMDO como herramienta informática para articular el aprendizaje, puesto que el sitio web cumple con las especificaciones 
necesarias para su implementación. Adicionalmente, y de forma simultánea, se utilizó Google Classroom (que es un sistema gestor de aprendizaje), plataforma educativa que sirvió como complemento para fortalecer las actividades propuestas.

Figura 6. Bloque PACIE - Datos informativos. Fuente: Elaboración propia

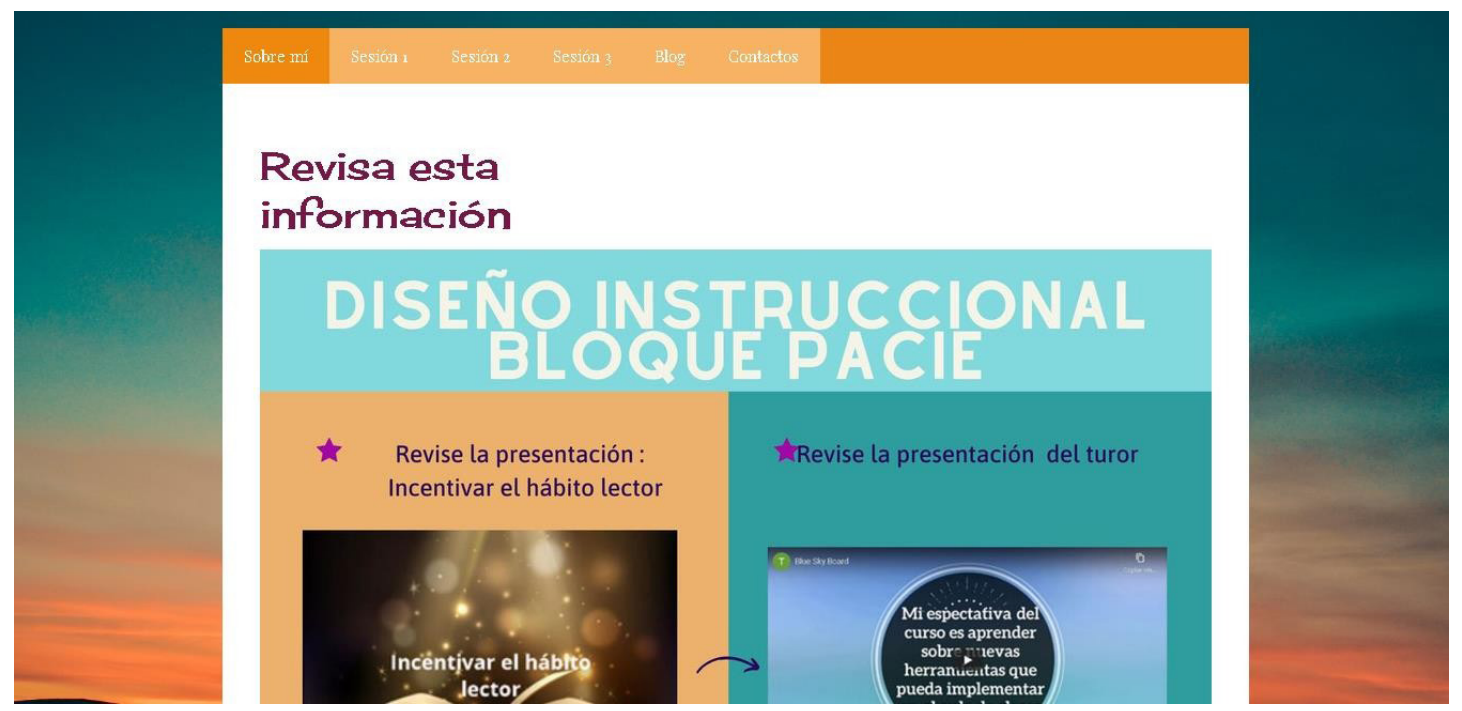

Esta manera de interactuar con los estudiantes fue innovadora y potenció sus habilidades. Cada actividad estuvo pensada de tal manera que, paso a paso, se pueda lograr el desarrollo de un sólido hábito lector. Adicionalmente, se buscó que los estudiantes fortalezcan, además de sus habilidades de lectoescritura, otras que les servirán tanto en su desempeño profesional, como en su vida cotidiana.

Una de las principales problemáticas era que los estudiantes mencionaron que no tenían tiempo para leer, por lo que en las primeras sesiones se puso mucho empeño para darles a conocer los beneficios de la lectura. Simultáneamente, se les indicó la manera en la que deben planificar sus actividades para que tengan el espacio y tiempo suficiente para leer.

Otro de los aspectos importantes que se deben recalcar es que la organización y distribución del tiempo son algo factores que pasan inadvertidos en la vida de los estudiantes. Enseñarles a planificar y organizar su tiempo es parte primordial dentro del proyecto. La mayoría de estudiantes no tenían idea de cómo organizar sus actividades, por eso se realiza una actividad introductoria en la que se les indica lo importante de distribuir su tiempo y luego ellos, en una actividad práctica, desarrollaron su planificación. De esta manera pudieron visualizar de manera clara cuánto tiempo se desperdicia y lo podrían ocupar en actividades relacionadas con la lectura. 
Los estudiantes, al poner en práctica las técnicas y consejos compartidos en el sitio web, afirmaron que es necesario organizarse para conseguir los objetivos propuestos y, que es posible tener el tiempo suficiente para ejecutar todas sus actividades, incluyendo la lectura. Se dieron cuenta de la importancia de planificar su tiempo, de cuanto se desperdicia; además que existen actividades que se pueden combinar con audiolibros, lo cual es una buena manera de adentrarse en la actividad lectora.

\section{DISCUSIÓN}

La tecnología, como apoyo para fortalecer capacidades en los estudiantes, fue la idea principal de la investigación realizada. Aclarar algunos paradigmas que aún se sostienen con respecto a que la tecnología es un distractor, se convierte en algo fundamental para la aplicación de las TIC en el campo educativo; existen variadas herramientas y actividades que se apoyan de la tecnología para potenciar habilidades importantes en los estudiantes. Lo importante es saber qué estrategia se usa con los estudiantes, buscando herramientas que sean fáciles de usar y que apoyen las distintas maneras de aprender.

Calero et al. (2010) mencionan que las TIC:

Aportan indicios que nos aproximan a la hipótesis inicial, confirmando parcialmente las creencias que el profesorado tiene sobre el significado de las tecnologías en la educación se encuentran relacionadas con su frecuencia y uso, sus percepciones sobre la integración organizativa y curricular, y con su formación y capacitación tecnológica (p. 1).

Esto quiere decir que, en la actualidad, aún se manifiesta cierta resistencia en cuanto al uso de la tecnología, los docentes en muchos de los casos sienten que la tecnología es un distractor, pero hay que saber utilizar y manejar la tecnología de la manera adecuada, buscar la estrategia adecuada para que la tecnología sea un apoyo y no un distractor, paulatinamente y con la ayuda de dichas iniciativas, aquella resistencia se ira disipando.

Buscar actividades que no sean las que siempre se han utilizado es otro factor que se tomó en cuenta para realizar el proyecto. Se hizo una amplia investigación en cuanto a la aplicación de actividades recreativas, pero que a la par fomenten la lectura. Algo interesante que tuvieron dichas actividades es que, para poder hacerlas, es necesario entender lo que se está exponiendo en cada una de ellas, entender por qué se realiza una actividad fomenta un grado 
de conciencia de las actividades. Es importante lo que menciona Zayas (2008) con respecto a lo que se dice anteriormente:

El libro de texto no puede ser la única fuente de lectura; el profesor debe conocer los distintos géneros, y se precisan procedimientos didácticos específicos y estrategias como crear metas de lectura, hacer predicciones textuales u operar con la estructura del texto. En definitiva, se trata de conseguir que leer tenga un sentido para el alumnado” (p. 71).

En base a esto, es importante buscar la manera de captar la atención del estudiante hacia la lectura y en la página web JIMDO se buscaron, desde el inicio, diferentes alternativas para lograrlo.

Ante los resultados de la investigación, se determinó que, por las condiciones socioeconómicas de los estudiantes, muchos de ellos no tienen el acceso a libros, lo que dificulta aún más gestionar la lectura en su contexto. El tener un acceso a repositorios de libros gratuitos facilita, de cierta manera, desarrollar un hábito lector. Cordón (2018) afirma que "nunca han concitado tanta atención los libros y la lectura como ahora, en que los cambios tecnológicos y la posibilidad de una migración similar a la producida en otros géneros”. En otras palabras, es necesario dar el salto a la era digital y el ámbito educativo es el más indicado para hacerlo, eliminando o acortando las limitaciones que puedan existir en los diferentes contextos que atañen a los estudiantes.

\section{CONCLUSIONES}

Es prudente determinar que, es de suma importancia fomentar el hábito lector desde una temprana edad; sin embargo, existe un reducido número de estudiantes en el nivel de educación superior que cumplen con dicha condición. Por ello, las actividades realizadas con los estudiantes que tenían una cultura lectora escasa, se direccionaron en base al contexto en el que puedan desarrollarse adecuadamente. Esto significa que fue muy importante analizar la situación de los estudiantes para desarrollar de mejor manera sus habilidades de lectoescritura.

Se propuso actividades diferentes e innovadores para afianzar el hábito de lectura en estudiantes de nivel superior. Además, se dio gran valor a plataformas tecnológicas como JIMDO y Google Classroom, las cuales son de fácil acceso y gratuitas. La incorporación de herramientas tecnológicas ayudó al desarrollo de distintas habilidades en los estudiantes, quienes lograron reforzar sus destrezas en varias áreas de conocimiento. 
También es muy importante tener en cuenta las necesidades de los estudiantes y el contexto que los rodea. En base a ello, se elaboraron las estrategias metodológicas que fomentaron el fortalecimiento del hábito de lectura y demás habilidades relacionadas.

Es importante realizar un diagnóstico al inicio de cada periodo estudiantil y no dejar este proceso cuando se inicia la etapa universitaria, pero además también es adecuado implementar una parte en la que se determine que hábitos en cuanto a lectura poseen los estudiantes, en base a esto trabajar de manera conjunta con la materia a desarrollarse.

Otro factor que se debe tener en cuenta es el desarrollo de habilidades tecnológicas que tenga el docente, ya que será quien impulse el uso de la plataforma para generar el hábito lector en los estudiantes.

Finalmente, es fundamental fomentar y desarrollar un hábito de lectura desde edades tempranas y a lo largo de toda la vida académica de los estudiantes, pero no hay que dejar de hacerlo en la educación superior pues el hecho de saber leer y escribir de forma adecuada es un requisito indispensable dentro del ámbito laboral.

\section{REFERENCIAS BIBLIOGRÁFICAS}

Abreu, J. (2014). El Método de la Investigación Research Method. Daena: International fournal of Good Conscience, 9 (3), pp. 195-204. http://www.spentamexico.org/v9-n3/A17.9(3)195-204.pdf

Álvira, M. (2011). La encuesta: una perspectiva general metodológica. Madrid, España: Centro de Investigaciones Sociológicas.

Calero, M.; Carles, R.; Mata, S. \& Navarro, E. (2010). Diferencias en habilidades y conducta entre grupos de preescolares de alto y bajo rendimiento escolar. RELIEVE, 16 (2), pp. 1-17. https://ojs. uv.es/index.php/RELIEVE/article/view/4152/3767

Camacho, P. (2012). Aparición de P.A.C.I.E. [Archivo PDF]. http://www.fatla.org/peter/pacie/correcto/ doc/pacie.pdf

Caldera de Briceño, R.; Escalante de Urrecheaga, D.; \& Terán de Serrentino, M. (2010). Práctica pedagógica de la lectura y formación docente. Revista de Pedagogía, 31 (88), pp. 15-38. https://www. redalyc.org/pdf/659/65916617002.pdf

Carlino, P. (2003). Leer textos científicos y académicos en la educación superior: obstáculos y bienvenidas a una cultura nueva. UNI-PLURI/VERSIDAD, 3(2), pp. 17-23. https://revistas.udea.edu.co/ index.php/unip/article/download/12289/11146/ 
Carlino, P. (2005). Escribir, leer, y aprender en la universidad. Una introducción a la alfabetización académica. Buenos Aires, Argentina: Fondo de cultura económica. https://www.aacademica.org/ paula.carlino/3.pdf

Cedeño, N. (agosto de 2012). LA INVESTIGACIÓN MIXTA, ESTRATEGIA ANDRAGÓGICA FUNDAMENTAL PARA FORTALECER LAS CAPACIDADES INTELECTUALES SUPERIORES. RES NON VERBA, s.v. (s.n.), pp. 17-36. https://biblio.ecotec.edu.ec/revista/edicion2/LA\%20 INVESTIGACI\%C3\%93N\%20MIXTA\%20ESTRATEGIA\%20ANDRAG\%C3\%93GICA\%20FUNDAMENTAL.pdf

Cordón, J. (2018). Libros electrónicos y lectura digital: los escenarios del cambio. Palabra Clave (La Plata), 7 (2), pp. 1-6. https://www.palabraclave.fahce.unlp.edu.ar/article/view/PCe044/9322

Diario Semana. (29 de septiembre de 2017). La mayoría de latinoamericanos culminan la secundaria sin saber leer bien. Semana. https://www.semana.com/educacion/articulo/unesco-niveles-delectura-en-america-latina/541971/

González, A. \& Gallardo, T. (2007). Investigación educativa. Arequipa: Editorial Universidad Nacional "San Agustín". Citado en: http://scielo.sld.cu/scielo.php?script=sci_arttext\&pid $\underline{=S 1990-86442019000500444}$

Hernández, I. (2013). FLORIDI: INFORMACIÓN Y FILOSOFÍA. THÉMATA, s.v. (49), pp. 127-142. https://www.academia.edu/7412685/Floridi_Informaci\%C3\%B3n_y Filosof\%C3\%ADa Revista Themata public

Instituto Nacional de Estadísticas y Censos. (2013). 5 de cada 10 ecuatoriano dedican al menos una hora a la semana a la lectura. https://www.ecuadorencifras.gob.ec/5-de-cada-10-ecuatorianodedican-al-menos-una-hora-a-la-semana-a-la-lectura/

León, J. (27 de junio de 2018). La lectura como hábito y herramienta para el desarrollo [Entrada de Blog]. https://www.blog.estadonacion.or.cr/index.php/25-la-lectura-\%20como-habito-y-herramienta-para-el-desarrollo

Oñate, L. (2009). La metodología PACIE. Quito, Ecuador: Fundación para la Actualización Tecnológica de Latinoamérica. https://www.academia.edu/download/50132854/La-Metodologia-Pacie.pdf

QuestionPro.com (24 de abril de 2020). Muestreo intencional. Características y ejemplos [Entrada de Blog]. https://www.questionpro.com/blog/es/muestreo-no-probabilistico/

Rodríguez, G.; García, E. \& Ibarra, M. (2013). SECUENCIA ERCA. MODELO DE SECUENCIA DE APRENDIZAJE PARA EL APRENDIZAJE ADULTO [Archivo PDF]. https://edufisicascjb.jimdofree.com/ app/download/11164520028/ERCA+\%20UCA.pdf?t=1478619478\&mobile=1 
Sabori, L. (13 de marzo de 2009). AUSBEL [Entrada de Blog]. http://teoriasunikino.blogspot.com/2009/03/ ausbel.html

Siemens, G. (2004). ¿Qué es el Conectivismo?: Teoría del Aprendizaje Para la Era Digital [Entrada de Blog]. Citado en: https://eduarea.wordpress.com/2014/03/19/que-es-el-conectivismo-teoriadel-aprendizaje-para-la-era-digital/

Trejo, A. \& Alarcón, L. (2006). Estrategias de lectura en la universidad. Graffylia: Revista de la Facultad de Filosofía y Letras, s.v. (6), pp. 30-38. https://filosofia.buap.mx/Graffylia/6/30.pdf

Zayas, F. (2008). Qué hacer para enseñar a leer y a entender mejor. (pp. 70-73). Citado en: https://dialnet. unirioja.es/servlet/articulo?codigo $=2666556$ 